\title{
Role of echocardiographic left ventricular mass and carotid intima-media thickness in the cardiovascular risk assessment of asymptomatic patients with type 2 diabetes mellitus
}

\author{
K. K. Poppe, ${ }^{1,2}$ G. A. Whalley, ${ }^{1,3}$ J. B. Somaratne, ${ }^{4}$ S. Keelan, ${ }^{5}$ W. Bagg, ${ }^{1}$ C. M. Triggs ${ }^{2}$ and R. N. Doughty ${ }^{1}$ \\ 'Department of Medicine and ${ }^{2}$ Department of Statistics, The University of Auckland and ${ }^{3}$ Faculty of Medical Imaging, Unitec, Auckland, New Zealand, \\ and ${ }^{4}$ Cardiology Department, St Vincent's Hospital, Melbourne and ${ }^{5}$ Sir Charles Gairdner Hospital, Perth, Australia
}

\begin{abstract}
Key words
risk assessment, ultrasonography, carotid arteries, left ventricular hypertrophy, type 2 diabetes mellitus.

\section{Correspondence \\ Katrina Poppe, Department of Medicine, The University of Auckland, Private Bag 92019, \\ Auckland 1142, New Zealand. \\ Email:k.poppe@auckland.ac.nz}

Received 8 April 2010; accepted 8 June 2010

doi:10.1111/j.1445-5994.2010.02305.x

\begin{abstract}
Background: Standard cardiovascular (CV) risk assessment may underestimate risk in people with type 2 diabetes mellitus (T2DM). Cardiac and vascular imaging to detect subclinical disease may augment risk prediction. This study investigated the association between CV risk, left ventricular hypertrophy (LVH) and carotid intima-media thickness (CIMT) in patients with T2DM free of CV symptoms.

Methods: People with T2DM without known CV disease were recruited from general practice. The 5-year risk of $\mathrm{CV}$ events was calculated using an adjusted Framingham equation and the prevalence of LVH and abnormal CIMT across bands of CV risk assessed. In those at intermediate risk, the number needed to scan (NNS) to reclassify one person to high risk was calculated across the group and compared in those above and below 55 years. The association between LV mass and CIMT was also assessed.

Results: Mean age 57 years (SD 1 1), 51\% female. Median 5-year CV risk 14.3\% (interquartile range 10.3, 19.5), 51\% had LVH (American Society of Echocardiography criteria) and $31 \%$ an abnormal CIMT (age and sex criteria). In the $52 \%$ at intermediate risk, $37 \%$ had LVH and $36 \%$ an abnormal CIMT. The NNS was 1.7 using both imaging techniques, 2.7 using cardiac imaging alone or 2.8 using vascular imaging alone. Almost twice as many people $>55$ years had an abnormal CIMT than those $<55$ years.

Conclusions: Cardiac and vascular imaging to detect subclinical disease can be used to augment prediction of CV risk in people with T2DM at intermediate risk. The value of reclassifying risk is as yet unproven and requires outcome data from intervention studies.
\end{abstract}

\section{Introduction}

Cardiovascular disease (CVD) is the leading cause of mortality and morbidity in people with type 2 diabetes mellitus (T2DM). ${ }^{1}$ Hypertension, dyslipidaemia and other $\mathrm{CV}$ risk factors contribute to the increased CVD risk in patients with T2DM. Standard CV risk assessment, incorporating conventional risk factors such as age, blood pressure (BP), smoking status and cholesterol, may

Funding: The study was supported by a Project Grant from the National Heart Foundation of New Zealand and the Cardiovascular Ultrasound Laboratory has received financial support from the New Zealand Lotteries Board. K. K. P. is the recipient of a postgraduate scholarship from the National Heart Foundation of New Zealand. J. B. S. was supported by the Douglas Goodfellow Medical Research Fellowship, the Auckland Medical Research Foundation and the Green Lane Research and Educational Fund.

Conflict of interest: None. underestimate risk. CV risk is a continuum and improving risk estimation, especially in those assessed as having intermediate risk, may help to identify those patients with T2DM that would benefit most from more intensive CV management.

Left ventricular hypertrophy (LVH) and carotid intimamedia thickness (CIMT) are markers of subclinical CVD and may reflect the accumulated effect of exposure to risk factors which are unrecognized or underrepresented by classic risk equations. $\mathrm{LVH}$ is associated with CV morbidity and mortality, independent of established risk factors such as age, sex and diabetes. ${ }^{2,3}$ There is a positive, linear relationship between $\mathrm{LV}$ mass and $\mathrm{CV}$ events in the general population ${ }^{4}$ and among patients with essential hypertension. ${ }^{5}$ Diabetes is associated with $\mathrm{LVH}^{6}$ and a large proportion of patients with T2DM without known CVD have $\mathrm{LVH}^{7}$. CIMT is a marker of atherosclerotic burden and has previously been associated with LV mass. ${ }^{8-10}$ The relationship between CIMT and the 
development of CVD has led to it being recommended as an adjunct to risk prediction particularly in those estimated to be at low to intermediate CVD risk. ${ }^{1-14}$ Previous studies suggest that $20-30 \%$ of people considered at low or intermediate risk by Framingham risk assessment equations are reclassified to high risk on the basis of abnormal CIMT. ${ }^{15,16}$ However, the role of CV imaging for reclassification of risk in patients with T2DM without known CVD has not been tested, and thus remains uncertain.

The aim of this study was to assess the association between CV risk, LVH and CIMT in patients with T2DM without known CVD.

\section{Methods}

\section{Study population}

The second Natriuretic Peptides in the Community study was a prospective cross-sectional study in New Zealand that sought to compare the value of NT-proBNP with electrocardiography (ECG) in the detection of LVH among patients in primary care with T2DM and no known CVD. The methods and results have been described previously. ${ }^{17}$ The study was approved by the local ethics committee, and informed consent was obtained from all subjects. Briefly, ethnically diverse participants with T2DM for $>5$ years and/or on treatment for T2DM, but without known CV, cerebrovascular or peripheral arterial disease were recruited from general practice in 2006-2007. CV risk factors were prevalent: $60 \%$ had hypertension, $70 \%$ had dyslipidaemia and $56 \%$ met American Society of Echocardiography (ASE) sexspecific criteria for LVH. The study found that while NT-proBNP was superior to ECG for the detection of LVH, it was unsuitable as a screening tool for LVH in patients with T2DM. The present study includes 298 patients in whom LV mass, CIMT and CV risk could be assessed.

\section{Estimation of cardiovascular risk}

The 5-year absolute risk of CVD events was estimated on the basis of age, sex, smoking, diabetes, BP and total : high-denisty lipoprotein (HDL) cholesterol using a Framingham equation. ${ }^{18}$ Variable definitions differed from those in the equation in that smoking was defined as being a current smoker only and did not include smoking in the past year, and ECG-defined LVH was not included as it is not routinely used for risk assessment in clinical practice.

To improve calibration of the Framingham equation in the New Zealand population, the New Zealand Guidelines Group (NZGG) recommends that $5 \%$ be added to the absolute risk estimate for one of: a family history of premature ischaemic CVD or ischaemic stroke in a firstdegree relative, being of a high-risk ethnicity (Maori, Pacific, Indian subcontinent), or having advanced diabetes (of more than 10 years duration or HbAlc consistently greater than $8 \%$ or the presence of microalbuminuria). ${ }^{19}$ A single adjustment is made for people with more than one of the above criteria. In those with a risk estimate of less than $15 \%$, but with extreme values of cholesterol (total or total : HDL $\geq 8$ ) or $\mathrm{BP}(\geq 170 \mathrm{mmHg}$ systolic or $100 \mathrm{mmHg}$ diastolic), the NZGG recommend they be classified as being at 15\% risk. The adjustment for ethnicity has subsequently been validated in a review of over 27000 patients. ${ }^{20}$ Low risk is defined as a 5 -year absolute risk of a CVD event of less than 5\%, intermediate risk is $5-15 \%$ and high risk is $\geq 15 \%$.

\section{Left ventricular hypertrophy}

All patients underwent a transthoracic echocardiogram (Philips iE33, Bothell, WA, USA). LV mass was calculated from m-mode images in accordance with the ASE recommendations ${ }^{21}$ and indexed to body surface area. LVH was defined by both the ASE (women $>95 \mathrm{~g} / \mathrm{m}^{2}$, men $\left.>115 \mathrm{~g} / \mathrm{m}^{2}\right)^{21}$ and the European Society of Hypertension $(\mathrm{ESH}) \quad\left(\text { women }>110 \mathrm{~g} / \mathrm{m}^{2} \text {, men }>125 \mathrm{~g} / \mathrm{m}^{2}\right)^{22}$ guidelines.

\section{Carotid intima-media thickness}

Carotid artery imaging was performed using a Sonosite Micromaxx (Bothell, WA, USA). The CIMT of the far wall of the right distal common carotid artery was manually measured from B-mode images in accordance with ASE recommendations. ${ }^{23}$ The reference range used to define abnormality was selected to match the method of measurement used in the study. Therefore, an abnormal CIMT was defined as above the 75th centile of age- and sex-specific ranges developed in the Atherosclerosis Risk in the Community (ARIC) study. ${ }^{24,25}$

\section{Approach to analysis}

The prevalence of LVH and abnormal CIMT across increasing bands of risk were presented visually as barplots, with the trend in proportions assessed using linear regression. In those at intermediate risk, the number needed to scan (NNS) to reclassify one person was calculated as the number at risk divided by the number that would be reclassified by one or both of the imaging techniques. The degree of overlap in risk reclassification by the presence of LVH or an abnormal CIMT is presented visually using Venn diagrams. These assessments were 


\begin{tabular}{|c|c|c|c|c|c|c|}
\hline & \multicolumn{6}{|c|}{ 5-year risk of CVD (\%) } \\
\hline & All & $<5$ & $5-10$ & $10-15$ & $15-20$ & $>20$ \\
\hline$n(\%)$ & 298 & $4(1)$ & $61(20)$ & $93(31)$ & $68(23)$ & $72(24)$ \\
\hline \multicolumn{7}{|l|}{ Diabetes } \\
\hline Diabetes duration, years & $6.0(3.3,11.0)$ & $5.5(3.8,7.8)$ & $5.0(2.0,8.0)$ & $5.2(3.0,8.3)$ & $6.0(3.0$ 12.0) & $10.0(6.0,14.5)$ \\
\hline $\mathrm{HbA} 1 \mathrm{c}, \%$ & $7.1(6.4,8.3)$ & $6.4(6.0,6.9)$ & $6.8(6.1,8.1)$ & $7.1(6.5,8.1)$ & $7.1(6.5,8.9)$ & $7.5(6.6,8.7)$ \\
\hline \multicolumn{7}{|l|}{ Risk factors } \\
\hline Female, $n(\%)$ & $151(51)$ & $1(25)$ & $35(57)$ & $42(45)$ & $41(60)$ & $32(44)$ \\
\hline Age, yearrs & $57(11)$ & $50(7)$ & $48(9)$ & $57(8)$ & $59(10)$ & $64(10)$ \\
\hline $\mathrm{SBP}, \mathrm{mmHg}$ & $135(18)$ & $120(12)$ & $121(12)$ & $130(9)$ & $143(17)$ & $147(20)$ \\
\hline $\mathrm{DBP}, \mathrm{mmHg}$ & $82(11)$ & $78(10)$ & $78(10)$ & $80(8)$ & $85(1)$ & $84(12)$ \\
\hline Total : HDL & $3.6(1.0)$ & $2.9(0.6)$ & $3.2(0.9)$ & $3.5(1.1)$ & $3.7(1.0)$ & $4.0(1.0)$ \\
\hline Smoking, $n(\%)$ & $47(16)$ & 0 & $8(13)$ & $7(8)$ & $13(19)$ & $19(26)$ \\
\hline \multicolumn{7}{|l|}{ Imaging } \\
\hline LV mass, g & $211(166,258)$ & $250(219,252)$ & $176(141,212)$ & $195(161,243)$ & $228(179,266)$ & $246(210,287)$ \\
\hline LVH by ASE, $n(\%)$ & $153(51)$ & $2(50)$ & $14(23)$ & $43(46)$ & $39(57)$ & $55(76)$ \\
\hline LVH by ESH, $n(\%)$ & $105(35)$ & $2(50)$ & $8(13)$ & $26(28)$ & $29(43)$ & $40(56)$ \\
\hline CIMT, mm & $0.75(0.65,0.86)$ & $0.60(0.58,0.62)$ & $0.69(0.62,0.76)$ & $0.74(0.6,0.87)$ & $0.75(0.66,0.86)$ & $0.81(0.73,0.92)$ \\
\hline Abnormal CIMT, $n(\%)$ & $92(31)$ & 0 & $27(44)$ & $29(31)$ & $20(29)$ & $16(22)$ \\
\hline
\end{tabular}

Values are presented as mean (standard deviation) or median (interquartile range) unless otherwise stated.

ASE, American Society of Echocardiography; CIMT, carotid intima-media thickness; CVD, cardiovascular disease; DBP, diastolic blood pressure; ESH, European Society of Hypertension; HDL, high-denisty lipoprotein; LVH, left ventricular hypertrophy; SBP, systolic blood pressure.

made in the whole cohort, and after dividing into groups above and below 55 years of age.

The relationship between CIMT and LV mass was assessed using scatterplots and Pearson's linear correlation coefficient. To assess the practical relevance of the association between CIMT and LV mass, 95\% prediction intervals were calculated on the basis of univariable and multiple linear regressions of LV mass on CIMT. In the full model, mass was adjusted for age (years), sex, height $(\mathrm{cm})$, systolic BP $(\mathrm{mmHg})$ and hypertensive treatment $(\mathrm{Y} / \mathrm{N})$.

All analyses were performed using $\mathrm{R}$ statistical software. ${ }^{26}$

\section{Results}

Variables required for calculation of risk, LV mass and CIMT were available in 298 subjects and their characteristics are summarized in Table 1. The mean age was 57 years (SD 11) and half were female. Median duration of diabetes was 6 years (interquartile range (IQR) 3.3, 10) and median HbAlc was 7.1\% (IQR 6.4, 8.3). Microalbuminuria was present in $25 \%(n=74)$ and macroalbuminuria in $11 \%(n=31)$.

\section{Whole group}

\section{Risk}

The majority of the study subjects $(87 \%, n=260)$ had $5 \%$ added to their Framingham risk estimate as recommended by the New Zealand Guidelines. Over two-thirds of these $(68 \%, n=178)$ moved up one risk band solely on the basis of their ethnicity, irrespective of the levels of any other factors. The median 5 -year risk of a CVD event was 14.3\% (IQR 10.3, 19.5). As expected, the mean value of each risk factor increased as risk increased, with those at more than $20 \%$ risk being predominantly male, older (mean age 64 years), hypertensive (mean BP 147/84 mmHg) and with elevated total : HDL cholesterol (mean 4.0).

Only four people were at $<5 \%$ risk. All were white, normotensive, non-smokers, with no family history of premature ischaemic CVD. Three were men aged $<50$ years, all had total : HDL cholesterol $<3.5$ and albumin creatinine ratio $<0.5$.

\section{Left ventricular hypertrophy and carotid intima-media thickness}

The prevalence of LVH was $51 \%$ by ASE criteria and 35\% by ESH criteria. As shown in Figure la and lb, the proportion of LVH by either criterion increased with increasing risk (average of $16 \%$ per risk band with ASE criteria; $13 \%$ per risk band with European Society of Cardiology (ESC) criteria) and the linear trend was significant $(P \leq$ 0.0001 for both).

The prevalence of an abnormal CIMT was $31 \%$ by ageand sex-specific criteria. The median CIMT increased as risk increased (Table 1); however, the proportion of those classified as having an abnormal CIMT by age- and sexspecific criteria decreased with increasing risk (Fig. lc). 

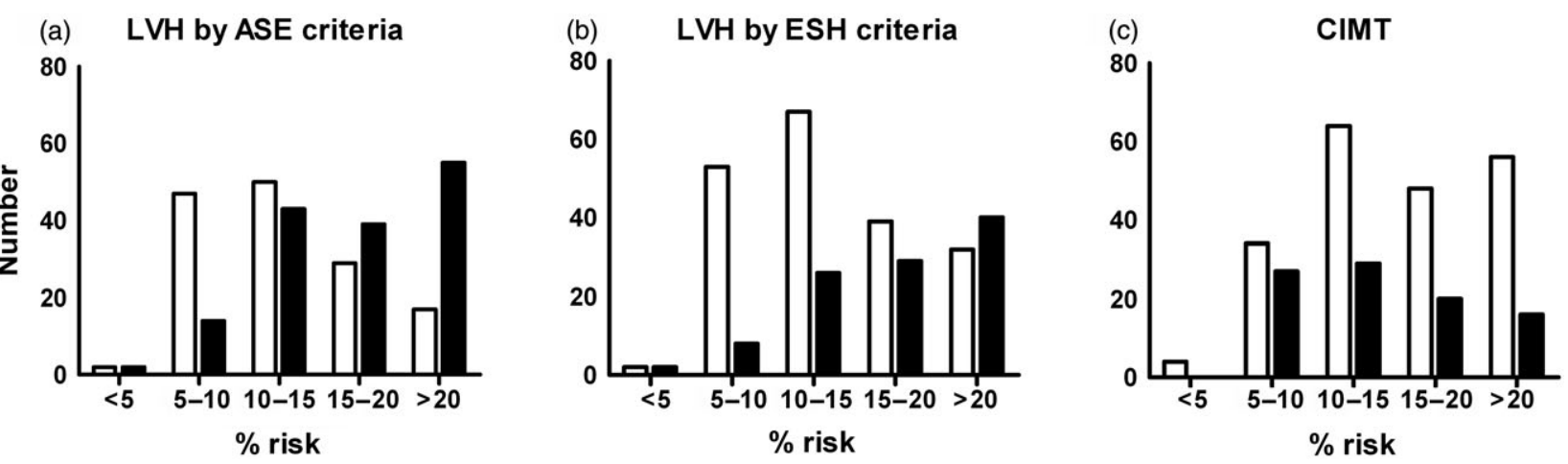

Figure 1 LVH and CIMT per band of 5-year cardiovascular risk. ASE, American Society of Echocardiography; CIMT, carotid intima-media thickness; ESH, European Society of Hypertension; light bar, within normal limits; LVH, left ventricular hypertrophy.

\section{Intermediate risk}

Fifty-two per cent of the group $(n=154)$ were at intermediate risk $(5-15 \%)$. Of these, $50 \%$ were female, mean age 53 years, mean BP 126/79 mmHg, total : HDL cholesterol was 3.4 and $10 \%$ were current smokers. LVH was present in $37 \%$ by ASE criteria or $22 \%$ by ESH criteria, and $36 \%$ had an abnormal CIMT.

The distributions of $\mathrm{LVH}$ and CIMT in this group are summarized in Figure 2. When $\mathrm{LVH}$ is defined using ASE criteria, $22 \%$ had LVH only and so would be reclassified to high risk on this basis, $21 \%$ would be reclassified on the basis of an abnormal CIMT only and 15\% had both conditions. Using both echocardiography and carotid imaging, the NNS to reclassify one person is 1.7 . If only one imaging technique is used, the NNS is 2.7 for echo and 2.8 for carotid imaging (however, more than $20 \%$ of people would not be reclassified using a single imaging technique).

When LVH is defined using ESC criteria, 12\% would be reclassified to high risk on the basis of LVH only, $26 \%$ on the basis of an abnormal CIMT only and 10\% had both conditions. Using both imaging modalities, the NNS is 2.1, with echo only is 4.5 and with carotid imaging only is 2.8 . Imaging with a single technique will not reclassify $26 \%$ and $12 \%$ respectively.

\section{Effect of age}

Age influences both CIMT and estimates of risk; therefore, the correlation between CIMT and risk is attenuated after adjustment for age, particularly in the higher risk bands. Figure 3 shows the effect of dividing the group at a nominal threshold of 55 years. In those aged less than 55 years, the prevalence of abnormal CIMT in people at intermediate $(5-15 \%)$ risk is almost twice that of those aged over 55 years ( $46 \%$ vs $24 \%$ ).
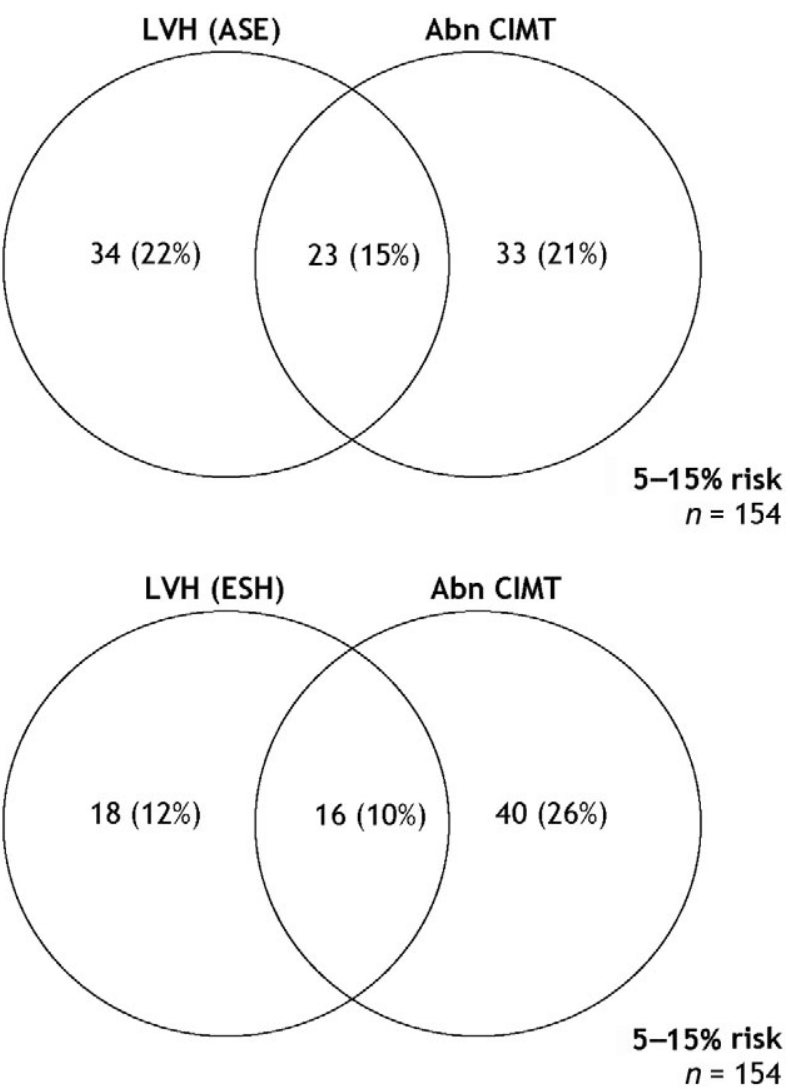

Figure 2 Proportion reclassified by one or both imaging modalities, in those at mild to moderate risk. Abn CIMT, abnormal carotid intima-media thickness; LVH (ASE), left ventricular hypertrophy as defined by American Society of Echocardiography criteria; LVH (ESH), left ventricular hypertrophy as defined by European Society of Hypertension criteria.

LV mass is not correlated to age and so is not adjusted for age when categorized to LVH. In those at intermediate risk, there is a greater difference in the proportion of LVH above and below 55 years using ASE than ESC criteria (Fig. 3). 
Under 55 years
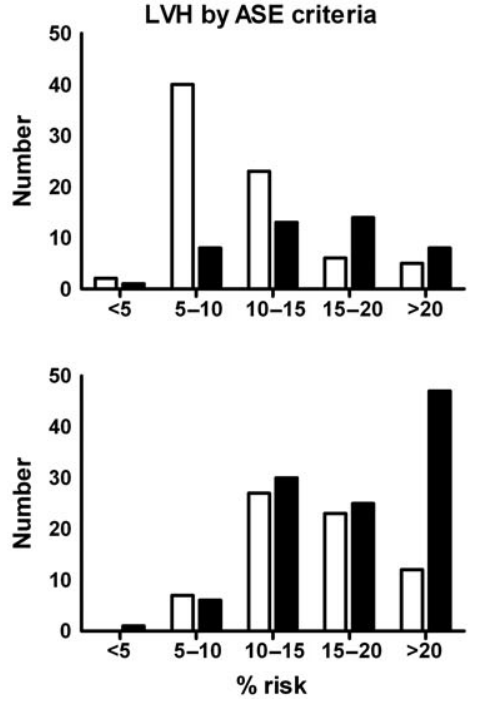
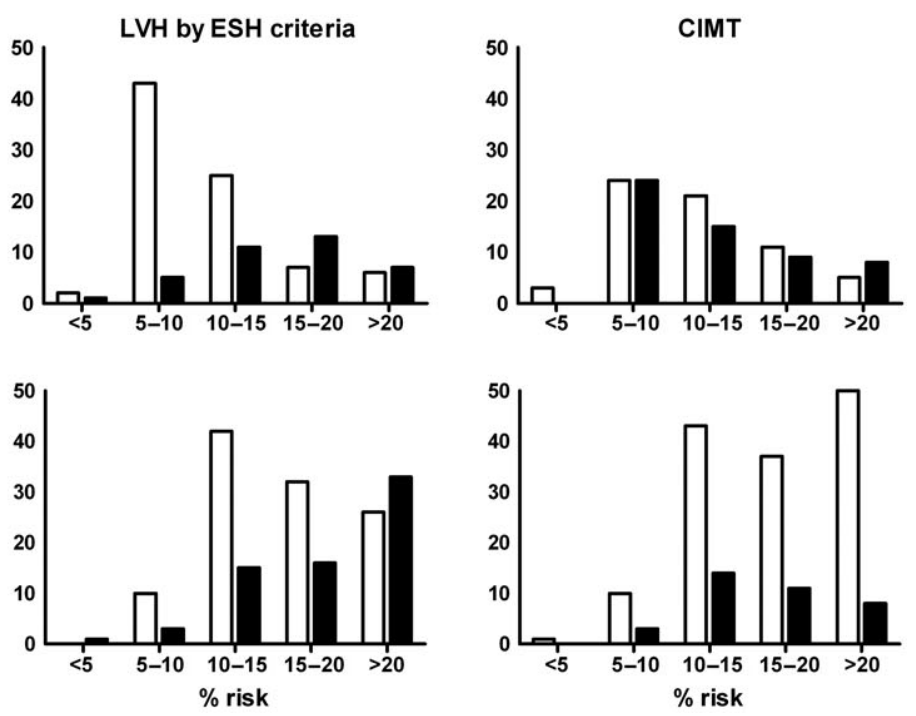

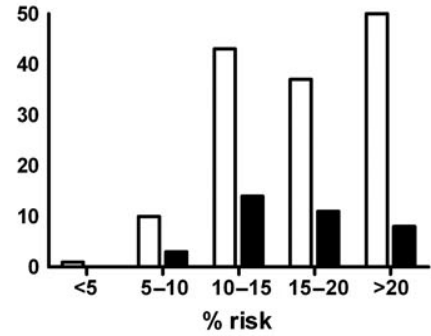

Figure 3 LVH and CIMT per band of 5-year cardiovascular risk, divided by age. ASE, American Society of Echocardiography; CIMT, carotid intima-media thickness; ESH, European Society of Hypertension; light bar, within normal limits; LVH, left ventricular hypertrophy.

\section{Left ventricular mass and carotid intima-media thickness}

The relationship between LV mass and CIMT is shown in Figure 4. The linear correlation coefficient $(r)$ is 0.22 (95\% CI $0.11,0.33)$. The correlation is significantly different from zero $(P=0.0001)$; however, the $95 \%$ prediction intervals for LV mass are extremely wide over the range of CIMT values, with little difference after adjusting CIMT for age, sex, height, systolic BP and hypertensive treatment (Fig. 4b). A CIMT of $0.63 \mathrm{~mm}$ will predict mass as being from 72 to $338 \mathrm{~g}$ (univariate) or 92 to $322 \mathrm{~g}$ (multivariate). Similarly, a CIMT of $0.98 \mathrm{~mm}$ will predict mass of 101 to $368 \mathrm{~g}$ (univariate) or 117 to $347 \mathrm{~g}$ (multivariate).

\section{Discussion}

In this study of a community cohort of people with T2DM and without symptomatic CVD, over half were at intermediate risk of a CV event in the next 5 years based on standard risk factors. Despite these patients having (a)

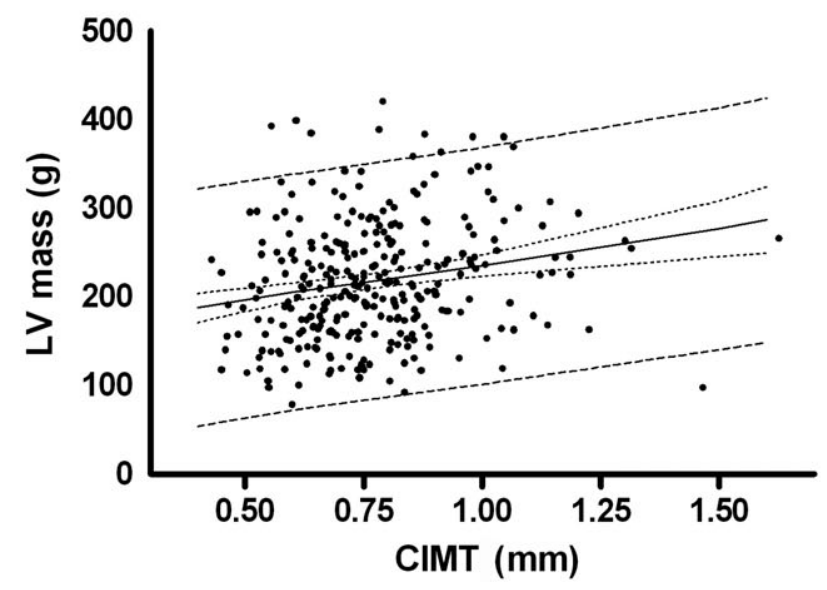

(b)

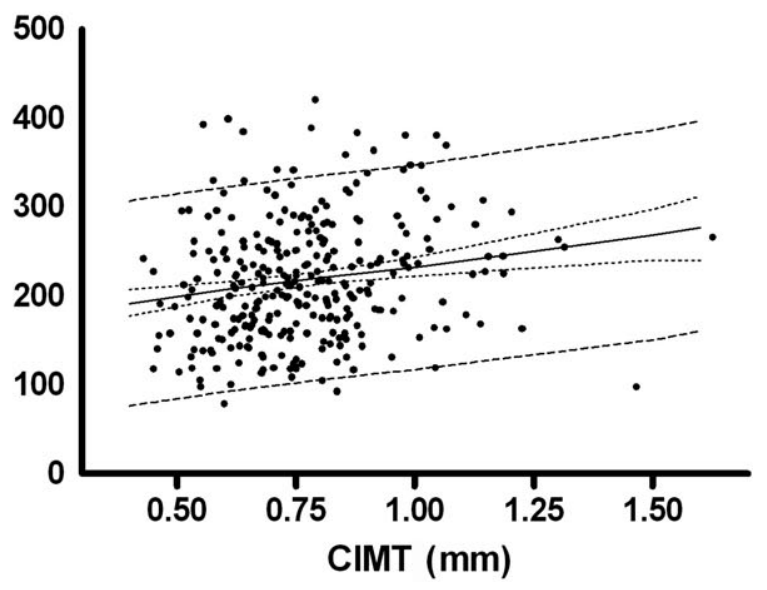

Figure 4 Scatterplots of CIMT and LV mass showing confidence and prediction intervals from (a) univariate and (b) multivariate analyses. CIMT, carotid intima-media thickness; dashed line, 95\% prediction interval; dotted line, 95\% confidence interval; LV, left ventricular; solid line, correlation. 
T2DM, there is uncertainty in CV and diabetes guidelines about how to manage CV risk. ${ }^{1,12,27}$ Consequently, improvement in risk prediction, especially among those in the intermediate-risk group, may provide more certainty on which to base appropriate management interventions. The results from the current study demonstrate that a combined imaging strategy, with cardiac and vascular imaging, may reclassify risk among those within the intermediate-risk group.

As both CIMT and LVH are markers of subclinical disease and are associated with CV events, it is relevant to consider the use of imaging techniques to augment risk prediction, especially in those at intermediate risk. ${ }^{11,12,23}$ Disease can occur in any part of the vascular system and so manifests as different events. Differing event rates and individual propensity to stroke, myocardial infarction (MI), or peripheral arterial disease are consistent with different mechanisms underlying the spectrum of CVD, and it would be unreasonable to expect one technique to identify all events for reclassification. Recent data support this concept; for example, the Multi-Ethnic Study of Atherosclerosis found that coronary artery calcium (CAC) score was more predictive of CHD and CVD compared with CIMT, which was more predictive of stroke, ${ }^{28}$ and recent genetic studies have identified a locus on chromosome 9p that is associated with $\mathrm{MI}$ and $\mathrm{CAD}$, and with CAC score, but not with CIMT. ${ }^{29}$

\section{Distribution of subclinical disease across risk categories}

In the current study, the prevalence of LVH within each risk band increased with increasing risk. Importantly, LVH was present at all levels of risk, and at least $20 \%$ of the group at intermediate risk would be reclassified to high risk on this basis. Conversely, using age- and sex-specific thresholds, the prevalence of an abnormal CIMT did not increase with increasing risk and may even decrease. Yet, unadjusted CIMT has been shown in this study and others $^{30,31}$ to increase with increasing risk. This relationship appears to be driven by the confounding effect of age: when the effect of age is removed (by categorizing to age-specific ranges), the relationship between CIMT and risk is attenuated. This is in contrast to LV mass, which is not affected by age, and in which the positive relationship with risk persists after categorization.

The effect of age is confirmed when stratifying the current data into two age bands, that is, above and below 55 years. The low prevalence of abnormal CIMT per risk band in those over 55 years shows that few people in this group would have risk status changed on the basis of an abnormal CIMT; however, LVH is prevalent and could be used. At least half of those $<55$ years have an abnormal
CIMT, across all risk groups, suggesting that CIMT may improve risk stratification in younger people. One potential limitation though is that the reference values for CIMT are mainly derived from patients aged $>45$ years, ${ }^{24}$ and thus the utility of CIMT in patients $<45$ years is uncertain.

\section{Intermediate risk}

Abnormal CIMT and LVH are found in a similar proportion of patients in the intermediate-risk group, although these are not the same individuals. Less than a quarter of those with either an abnormal CIMT or LVH have both abnormalities. The lack of overlap of the two conditions may represent different aspects of the risk profile of T2DM, or may represent different stages of disease development.

\section{Reclassification of risk}

The combined high prevalence of LVH and an abnormal CIMT in those at intermediate risk meant that the NNS to reclassify risk was very low (NNS $=1.7)$. Although $21-22 \%$ of people will not be reclassified if only one imaging technique is used (using ASE criteria), the NNS is still 2.8 with CIMT, and 2.7 with echocardiography. Incorporation of cardiac and/or carotid imaging in risk assessment requires consideration of the extra time and cost of these procedures, as well as the availability of technology and suitably qualified personnel. ${ }^{12}$ A targeted approach of measuring LV mass and CIMT in a diabetic clinic, for example, would minimize these costs. Although relatively low, the inter- and intra-individual variability of image acquisition and measurement are important considerations for use in individual patients. Automated border detection for measurement of CIMT is recommended to limit variability in measurement, ${ }^{23}$ although this is not consistently used. ${ }^{24}$

\section{Correlation between left ventricular hypertrophy and carotid intima-media thickness}

CIMT is associated with LV mass although the linear correlation $(r)$ between them is weak, especially in non-hypertensive subjects. Consistent with previous studies, ${ }^{8-10}$ we found the correlation coefficient to be significantly different from zero $(P=0.0001)$; however, the low value of the coefficient $(r=0.22)$ and wide prediction intervals show that it is not a clinically significant relationship. The lack of association between CIMT and LV mass, however, supports the principle that the two approaches assess different aspects of subclinical disease, 
and that use of both may improve the estimation of $\mathrm{CV}$ risk.

\section{Limitations}

Adjustments to the risk estimate that are currently recommended in New Zealand Guidelines had a significant effect on the distribution of risk in this study. Eighty-seven per cent of the cohort had their risk estimate adjusted upwards, two-thirds of which was due to being of a highrisk ethnicity regardless of any other reason for adjustment. Subsequent studies have shown that although the ethnicity adjustment is suitable, adjusting for family history is not. ${ }^{20}$ This may be due to a confounding effect between family history and ethnicity, which may also confound with the adjustments for advanced diabetes; however, these have not been addressed. Consequently, we cannot confirm the validity of non-ethnic adjustments.

The reference range used to define an abnormal CIMT impacts on the prevalence of disease. The thresholds used in this study were those developed from the ARIC cohort (imaged in 1987-1989). ${ }^{24}$ Older imaging systems will generally have poorer resolution than current systems, thus CIMT values measured now may be lower than those imaged on an older machine. Subsequently, the amount of abnormal CIMT in current images may be underestimated by using reference ranges formulated using older technology. ${ }^{23}$ This study included people of ethnicities at increased CV risk; therefore, thresholds of abnormal CIMT established in the ARIC cohort may not be optimal. However, in the absence of a local reference range being available, a range recommended by the ASE was used.
Of the range of imaging measurements that are available, LVH and CIMT were selected as they have a proven association with CV risk. The accuracy and reproducibility of echocardiographic LV mass may be less than that of cardiac magnetic resonance imaging (MRI); however, MRI is not widely available and would cost more than echocardiography in a population such as this.

\section{Conclusion}

Accurate risk stratification for patients with T2DM is important to assist with decisions regarding preventative interventions; yet, standard risk assessment is suboptimal in this group. Cardiac and vascular imaging, to detect subclinical disease, can be used to augment risk prediction. The value of such imaging is likely to be greatest among those at intermediate risk in whom management may be less clear. Detection of abnormal CIMT and the presence of LVH would reclassify up to $58 \%$ of patients at intermediate risk to high risk, but the value of reclassifying risk is as yet unproven, and there remains a need for outcome data from appropriately designed intervention studies demonstrating that treatment interventions will improve outcome.

\section{Acknowledgements}

We acknowledge the assistance of Mariska ter Bals, Georgina Dew, Gael Harrison, Ann Pearl, Gina Wadams, Helen Walsh and Andrew Yeadon, from the Faculty of Medical and Health Sciences at The University of Auckland, with data collection.

\section{References}

1 American Diabetes Association. Consensus development conference on the diagnosis of coronary heart disease in people with diabetes. Diabetes Care 1998; 21: 1551-9.

2 Levy D, Garrison RJ, Savage DD, Kannel WB, Castelli WP. Prognostic implications of echocardiographically determined left ventricular mass in the Framingham Heart Study. $N$ Engl J Med 1990; 322: 1561-6.

3 Gosse P. Left ventricular hypertrophy as a predictor of cardiovascular risk. J Hypertens 2005; 23 (Suppl): S27S33.

4 Vasan RS, Larson MG, Levy D, Evans JC, Benjamin EJ. Distribution and categorization of echocardiographic measurements in relation to reference limits: the framingham heart study: formulation of a height- and sex-specific classification and its prospective validation. Circulation 1997; 96: 1863-73.

5 Schillaci G, Verdecchia P, Porcellati C, Cuccurullo O, Cosco C, Perticone F. Continuous relation between left ventricular mass and cardiovascular risk in essential hypertension. Hypertension 2000; 35: 580-86.

6 Fang Z, Prins J, Marwick T. Diabetic cardiomyopathy: evidence, mechanisms, and therapeutic implications. Endocr Rev 2004; 25: 543-67.

7 Palmieri V, Tracy RP, Roman MJ, Liu JE, Best LG, Bella JN et al. Relation of left ventricular hypertrophy to inflammation and albuminuria in adults with type 2 diabetes: the Strong Heart Study (Epidemiology/Health Services/ Psychosocial Research). Diabetes Care 2003; 26: 2764.
8 Muiesan ML, Salvetti M, Zulli R, Pasini G, Bettoni G, Monteduro C et al. Structural association between the carotid artery and the left ventricle in a general population in Northern Italy: the Vobarno study. J Hypertens 1998; 16: 1805-12.

9 de Simone G, McClelland R, Gottdiener JS, Celentano A, Kronmal RA, Gardin JM. Relation of hemodynamics and risk factors to ventricular-vascular interactions in the elderly: the Cardiovascular Health Study. J Hypertens 2001; 19: 1893-903.

10 Cuspidi C, Mancia G, Ambrosioni E, Pessina A, Trimarco B, Zanchetti A et al. Left ventricular and carotid structure in untreated, uncomplicated essential hypertension: results from the Assessment Prognostic Risk Observational Survey (APROS). J Hum Hypertens 2004; 18: 891-96. 
11 Greenland P, Abrams J, Aurigemma GP, Bond MG, Clark LT, Criqui MH et al. Prevention Conference V: beyond secondary prevention: identifying the high-risk patient for primary prevention: noninvasive tests of atherosclerotic burden: Writing Group III. Circulation 2000; 101: el6-22.

12 NCEP. Third report of the National Cholesterol Education Program (NCEP) Expert Panel on Detection, Evaluation, and Treatment of High Blood Cholesterol in Adults (Adult Treatment Panel III) final report. Circulation 2002; 106: 3143-421.

13 Baldassarre D, Amato M, Pustina L, Castelnuovo S, Sanvito S, Gerosa L et al. Measurement of carotid artery intima-media thickness in dyslipidemic patients increases the power of traditional risk factors to predict cardiovascular events. Atherosclerosis 2007; 191: 403-8.

14 Junyent M, Zambón D, Gilabert R, Núñez I, Cofán M, Ros E. Carotid atherosclerosis and vascular age in the assessment of coronary heart disease risk beyond the Framingham Risk Score. Atherosclerosis 2008; 196: 803-9.

15 Grewal J, Anand S, Islam S, Lonn E. Prevalence and predictors of subclinical atherosclerosis among asymptomatic 'low risk' individuals in a multiethnic population. Atherosclerosis 2008; 197: 435-42.

16 Cuspidi C, Lonati L, MacCa G, Sampieri L, Fusi V, Michev I et al. Prevalence of left ventricular hypertrophy and carotid thickening in a large selected hypertensive population: impact of different echocardiographic and ultrasonographic diagnostic criteria. Blood Press 2001; 10: 142-9.

17 Somaratne JB, Whalley GA, Poppe KK, ter Bals M, Walsh H, Wadams G et al. $\mathrm{N}$-terminal pro-brain natriuretic peptide is more useful than electrocardiograms for detecting left ventricular hypertrophy in asymptomatic patients with type 2 diabetes mellitus from primary care. Heart Lung Circ 2008; 17(Suppl 3): S136.

18 Anderson K, Odell P, Wilson P, Kannel W. Cardiovascular disease risk profiles. Am Heart J 1991; 121: 293-8.

19 New Zealand Guidelines Group. New Zealand Cardiovascular Guidelines Handbook: A Summary Resource for Primary Care Practitioners, 2nd edn. Wellington: Ministry of Health; 2009.

20 Broad JB, Marshall RJ, Wells S, Kerr AJ, Riddell T, Jackson R. Are NZ Guideline adjustments to Framingham scores justified for CVD risk prediction? Heart Lung Circ 2007; 16: S9-S10.

21 Lang RM, Bierig M, Devereux RB, Flachskampf FA, Foster E, Pellikka PA et al. Recommendations for chamber quantification: A report from the American Society of Echocardiography's guidelines and standards committee and the Chamber Quantification Writing Group, developed in conjunction with the European Association of Echocardiography, a branch of the European Society of Cardiology. J Am Soc Echocardiogr 2005; 18 : 1440-63.

22 Mancia G, De Backer G, Dominiczak A, Cifkova R, Fagard R, Germano G et al. 2007 Guidelines for the management of arterial hypertension: The Task Force for the Management of Arterial Hypertension of the European Society of Hypertension (ESH) and of the European Society of Cardiology (ESC). $J$ Hypertens 2007; 25: 1105-87.

23 Stein JH, Korcarz C, Hurst RT, Lonn E, Kendall CB, Mohler E et al. Use of carotid ultrasound to identify subclinical vascular disease and evaluate cardiovascular disease risk: a consensus statement from the American Society of Echocardiography Carotid Intima-Media Thickness Task Force Endorsed by the Society for Vascular Medicine. J Am Soc Echocardiogr 2008; 21: 93-111.

24 Howard G, Sharrett AR, Heiss G, Evans GW, Chambless LE, Riley WA et al. Carotid artery intimal-medial thickness distribution in general populations as evaluated by B-mode ultrasound. ARIC Investigators. Stroke 1993; 24: 1297-304.

25 Redberg RF, Vogel RA, Criqui $\mathrm{MH}$, Herrington DM, Lima JAC, Roman MJ. Task force \#3 - what is the spectrum of current and emerging techniques for the noninvasive measurement of atherosclerosis? J Am Coll Cardiol 2003; 41: 1886-98.

26 R Development Core Team. R: A Language and Environment for Statistical Computing, 2.8.1 edn. Vienna, Austria: R Foundation for Statistical Computing; 2008.

27 ESC Guideline ATFM, Graham I, Atar D, Borch-Johnsen K, Boysen G, Burell G et al. European guidelines on cardiovascular disease prevention in clinical practice: executive summary: Fourth Joint Task Force of the European Society of Cardiology and Other Societies on Cardiovascular Disease Prevention in Clinical Practice (Constituted by representatives of nine societies and by invited experts). Eur Heart J 2007; 28: 2375-414.

28 Folsom AR, Kronmal RA, Detrano RC, O'Leary DH, Bild DE, Bluemke DA et al. Coronary artery calcification compared with carotid intima-media thickness in the prediction of cardiovascular disease incidence: the Multi-Ethnic Study of Atherosclerosis (MESA). Arch Intern Med 2008; 168: 1333-9.

29 Sharma K, Blaha MJ, Blumenthal RS, Musunuru K. Clinical and research applications of carotid intima-media thickness. Am J Cardiol 2009; 103: 1316-20.

30 Bots ML, Hoes AW, Koudstaal PJ, Hofman A, Grobbee DE. Common carotid intima-media thickness and risk of stroke and myocardial infarction: the Rotterdam Study. Circulation 1997; 96: 1432-7.

31 O'Leary DH, Polak JF, Kronmal RA, Manolio TA, Burke GL, Wolfson SK Jr. Carotid-artery intima and media thickness as a risk factor for myocardial infarction and stroke in older adults. $N$ Engl J Med 1999; 340: 14-22. 